\title{
The inhomogeneous submillimeter atmosphere of Betelgeuse
}

\author{
E. O’Gorman ${ }^{1}$, P. Kervella ${ }^{2,3}$, G. M. Harper ${ }^{4}$, A. M. S. Richards ${ }^{5}$, L. Decin ${ }^{6}$, M. Montargès ${ }^{7}$, and I. McDonald ${ }^{5}$ \\ 1 Dublin Institute for Advanced Studies, 31 Fitzwilliam Place, Dublin 2, Ireland \\ e-mail: ogorman@cp.dias.ie \\ 2 Unidad Mixta Internacional Franco-Chilena de Astronomía (CNRS UMI 3386), Departamento de Astronomía, \\ Universidad de Chile, Camino El Observatorio 1515, Las Condes, Santiago, Chile \\ 3 LESIA (UMR 8109), Observatoire de Paris, PSL Research University, CNRS, UPMC, Univ. Paris-Diderot, 5 place Jules Janssen, \\ 92195 Meudon, France \\ 4 Center for Astrophysics and Space Astronomy, University of Colorado, 389 UCB, Boulder, CO 80309, USA \\ 5 JBCA, Department Physics and Astronomy, University of Manchester, Manchester M13 9PL, UK \\ ${ }^{6}$ Institute of Astronomy, KU Leuven, Celestijnenlaan 200D B2401, 3001 Leuven, Belgium \\ 7 Institut de Radioastronomie Millimétrique, 300 rue de la Piscine, 38406 Saint-Martin d'Hères, France
}

Received 15 May 2017 / Accepted 6 June 2017

\begin{abstract}
The mechanisms responsible for heating the extended atmospheres of early-M spectral-type supergiants are poorly understood. So too is the subsequent role these mechanisms play in driving the large mass-loss rates of these stars. Here we present ALMA long (i.e., $\sim 16 \mathrm{~km})$ baseline $338 \mathrm{GHz}(0.89 \mathrm{~mm})$ continuum observations of the free-free emission in the extended atmosphere of the M2 spectral-type supergiant Betelgeuse. The spatial resolution of 14 mas exquisitely resolves the atmosphere, revealing it to have a mean temperature of $2760 \mathrm{~K}$ at $\sim 1.3 R_{\star}$, which is below both the photospheric effective temperature $\left(T_{\text {eff }}=3690 \mathrm{~K}\right)$ and the temperatures at $\sim 2 R_{\star}$. This is unambiguous proof for the existence of an inversion of the mean temperature in the atmosphere of a red supergiant. The emission is clearly not spherically symmetric with two notable deviations from a uniform disk detected in both the images and visibilities. The most prominent asymmetry is located in the north-east quadrant of the disk and is spatially resolved showing it to be highly elongated with an axis-ratio of 2.4 and occupying $\sim 5 \%$ of the disk projected area. Its temperature is approximately $1000 \mathrm{~K}$ above the measured mean temperature at $1.3 R_{\star}$. The other main asymmetry is located on the disk limb almost due east of the disk center and occupies $\sim 3 \%$ of the disk projected area. Both emission asymmetries are clear evidence for localized heating taking place in the atmosphere of Betelgeuse. We suggest that the detected localized heating is related to magnetic activity generated by large-scale photospheric convection.
\end{abstract}

Key words. stars: atmospheres - stars: evolution - stars: imaging - stars: individual: Betelgeuse - submillimeter: stars

\section{Introduction}

The mechanisms responsible for driving the relatively large mass-loss rates $\left(\dot{M} \sim 10^{-4}-10^{-6} M_{\odot} \mathrm{yr}^{-1}\right)$ of red supergiants (RSGs) remain poorly understood and the associated issues have long been highlighted (e.g., Holzer \& MacGregor 1985). RSGs have wind terminal velocities that are lower than their photospheric escape velocities (i.e., $v_{\infty} \lesssim 0.5 v_{\text {esc }}$ ) and so most of the energy deposited into the wind does not go into the wind kinetic energy but must go into heating the atmosphere and lifting the atmosphere out of the gravitational potential well. An important constraint on the wind-driving mechanism of RSGs is that most of the energy must be deposited within the subsonic wind region to have any affect on mass-loss rates; energy deposited beyond merely modifies the wind velocity (Hartmann \& MacGregor 1980; Holzer et al. 1983). Therefore, to gain insight into the mechanisms responsible for mass loss in RSGs, we need to study the atmosphere within the first few stellar radii (i.e., the extended atmosphere), where the wind gains most of its energy through heating and momentum deposition.

Progress in this field is currently being driven by observations from state-of-art facilities that can provide the necessary spatial resolution to study the extended atmospheres of the closest RSGs. The Hubble Space Telescope (HST) partially resolved the hot $(\sim 8000 \mathrm{~K})$ chromospheric gas around Betelgeuse ( $\alpha$ Ori, M2Iab) and showed that the star in the ultraviolet
(UV) appears about $2.5 \times$ larger than the optical photosphere (43 mas in the infrared H-band, Montargès et al. 2016) and even more extended in the Mg II H and K lines (Gilliland \& Dupree 1996). The signature of an unresolved UV-bright asymmetry was also found and was suggested to be associated with the pole of the star (Uitenbroek et al. 1998). Kervella et al. (2016) imaged inhomogeneous hot $\mathrm{H} \alpha$ emission mostly contained within $3 R_{\star}$ with SPHERE on the Very Large Telescope (VLT) while Harper \& Brown (2006) showed that the chromospheric gas decouples from co-rotation at about $3 R_{\star}$. Lim et al. (1998) used multi-wavelength, spatially resolved Very Large Array (VLA) radio observations to show that the hot chromospheric gas has a small filling factor and that the mean temperature at these radii is relatively cool $\left(T_{\text {gas }} \lesssim 3600 \mathrm{~K}\right)$. Indeed, the presence of a dense, cool (1500-2000 K) molecular layer (so-called "MOLsphere") is thought to exist between $\sim 1.3$ and $1.5 R_{\star}$ to explain the infrared spectra of early M supergiants (e.g., Tsuji 2000; Ohnaka et al. 2011). It appears that the extended atmospheres of RSGs exhibit complicated thermal structures.

For late-M RSGs like VY CMa, the dominant source of opacity at submm wavelengths is from dust grains (O'Gorman et al. 2015b). However, for the earlier M-type RSGs such as Betelgeuse the dominant source of opacity at submm wavelengths is from $\mathrm{H}^{-}$opacity; thermal free-free interactions between free electrons from photoionized metals and neutral 
hydrogen (Harper et al. 2001). At these wavelengths, the thermal source function is simply the Planck function in the RayleighJeans limit, which is linear with the gas (electron) temperature. The mean gas temperature can then be empirically estimated using the Eddington-Barbier relation if the emission is spatially resolved. If the thermal structure is inhomogeneous at small scales (i.e., much smaller than the restoring beam), the source function is an average of the temperatures; unlike the UV, where very small volumes of hot plasma can dominate the total emission.

\section{Observations and analysis}

Betelgeuse was observed with the Atacama Large Millimeter/submillimeter Array (ALMA) on 9 November 2015 during cycle 3 in band $7(275-373 \mathrm{GHz})$ using an array configuration with projected baselines ranging from 0.078 to $16.076 \mathrm{~km}$ (project code: 2015.1.00206.S, PI: P. Kervella). The observations lasted for $75 \mathrm{~min}$ with $61 \mathrm{~min}$ spent on source. A total of 47 antennas were used to make the final calibrated visibility dataset. The observations were performed using five spectral windows (spws). Two spws were centered at $330.65 \mathrm{GHz}$ and $332.55 \mathrm{GHz}$ and covered a total bandwidth of $0.94 \mathrm{GHz}$ and $1.88 \mathrm{GHz}$, respectively. The three other spws were centered at $343.19 \mathrm{GHz}, 345.15 \mathrm{GHz}$, and $345.80 \mathrm{GHz}$ covering a total bandwidth of $1.88 \mathrm{GHz}, 0.94 \mathrm{GHz}$ and $0.47 \mathrm{GHz}$, respectively.

The phase-reference source $\mathrm{J} 0552+0313$ was located $4.2^{\circ}$ from Betelgeuse and was used to derive time-dependent phase and amplitude corrections. It was observed in $18 \mathrm{~s}$ scans and bracketed 1.5 min scans of Betelgeuse. The quasar J0510+1800 was used for bandpass and absolute flux density calibration assuming a flux density at $339.5 \mathrm{GHz}$ of $3.0 \mathrm{Jy}$ and a spectral index of $\alpha=-0.25$. We estimate an absolute flux density uncertainty of $5 \%$ based upon the uncertainty of the catalog values due to variability at the time. J0605+0939 was observed every 12 minutes and was used to check the absolute position of Betelgeuse (see Harper et al., 2017). The data were calibrated with the ALMA calibration pipeline and manually inspected and imaged using CASA 4.5.0. The continuum emission was split out from the molecular line emission and the continuum channel widths were averaged to $125 \mathrm{MHz}$. A Gaussian profile was then fitted to the target in the initial continuum image to allow the target to be shifted to phase center. The total continuum bandwidth amounted to $4.1 \mathrm{GHz}$ at a center frequency of $338 \mathrm{GHz}$ $(\lambda=0.89 \mathrm{~mm})$. Continuum imaging is described in Appendix A. To analyze the self-calibrated visibilities directly, we used the UVMULTIFIT task version 2.2.0-r3 (Martí-Vidal et al. 2014).

\section{Results}

The Briggs-weighted (robust 0) continuum image is shown in Fig. 1. The restoring beam FWHM of $15 \times 13$ mas clearly resolves the continuum emission with approximately 50 restoring beams fitting within the $3 \sigma$ contours. The angular diameter of the emission at the $3 \sigma$ level is approximately 100 mas. The emission is clearly not axisymmetric with the peak emission notably located in the north-east (NE) quadrant (hereafter NEpeak). Moreover, the emission around the location of NE-peak itself is elongated. The more extended emission also deviates from circular symmetry with another notable asymmetry almost directly east of the center of emission (hereafter east-extension). The slightly higher spatial resolution images made using Briggs (robust -1) and uniform weighting yield almost an identical morphology as in Fig. 1, therefore we exclude them from any further analysis and discussion due to their lower $\mathrm{S} / \mathrm{N}$.

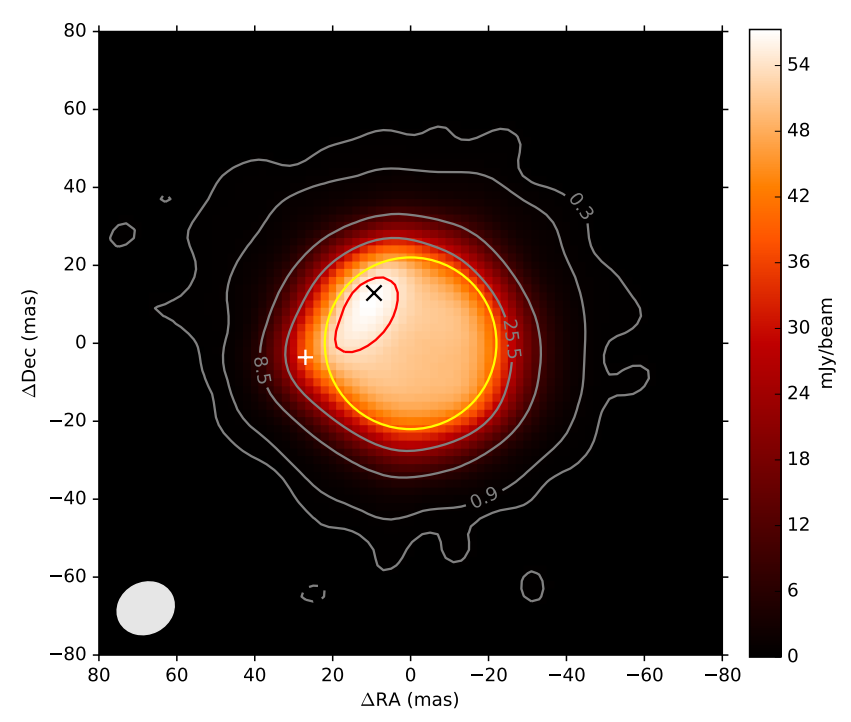

Fig. 1. ALMA $338 \mathrm{GHz}$ Briggs-weighted (robust 0) continuum image of Betelgeuse. Gray contours are set to $(-3,3,10,100,300) \times \sigma_{\text {rms }}$ where $\sigma_{\text {rms }}=85 \mu \mathrm{Jy}_{\text {beam }}^{-1}$ is the image rms noise. The numbers within the contours are the flux density values they represent in mJy. The largest contour is displayed in red for clarity at $54 \mathrm{mJy}$ or $635 \sigma_{\text {rms. }}$. The filled ellipse in the lower left corner is the full width at half maximum (FWHM) of the restoring beam and has dimensions $15 \times 13$ mas. The locations of the NE-peak and the east-extension are marked with " $\times$ " and "+", respectively. The yellow circle is the size of the infrared H-band photosphere from Montargès et al. (2016).

We fitted a number of models to the complex visibilities to accurately quantify the basic properties of the main emission features such as position, morphology, and flux density, and also search for any underlying concealed substructure that might not be obvious in the images. The models included a uniform elliptical disk either with or without a combination of point sources, elliptical or circular Gaussians, and an elliptical ring. After each fit, the residual images (i.e., data - model) were inspected to help guide the next possible improved fit. Some of these residual images are shown in Appendix B along with further details of the fitting process. The model that produced the lowest reduced chisquared statistic value as well as the lowest remaining residuals in the residual images is described in Table 1. It consists of one relatively large uniform elliptical disk that contains one resolved elliptical Gaussian at the position of the NE-peak, another resolved circular Gaussian at the position of the east-extension, and a thin (i.e., unresolved) elliptical ring outside of the uniform elliptical disk with the same center position and position angle (PA; measured east of north) as the main uniform elliptical disk.

The main uniform elliptical disk has a major axis of 57.8 mas, is mildly elongated with an axis ratio (major axis/minor axis) of 1.09 and has a brightness temperature, $T_{\mathrm{b}}$, of $2760 \pm 140 \mathrm{~K}$. The NE-peak was found to be highly elongated having a FWHM major axis of 19.8 mas and an axis ratio of 2.38. This elongation is clearly visible in both the continuum images and in the residual images where the component has not been subtracted (see the left panel of Fig. B.1). The total brightness temperature of the NE-peak was $3815 \pm 165 \mathrm{~K}$. This value is derived by adding its $T_{\mathrm{b}}$ value in Table 1 to the uniform elliptical disk value because the model fitting has shown the entire NE-peak lies directly on top of the uniform elliptical disk. The east-extension was characterized by a circular Gaussian profile centered almost exactly at the limb/edge of the main uniform elliptical disk. Its FWHM was 8.9 mas and its brightness temperature was $2260 \pm 135 \mathrm{~K}$. The total brightness temperature 
Table 1. Best-fit multi-component model to the ALMA band 7 continuum visibilities.

\begin{tabular}{cccccccc}
\hline \hline Component & $\begin{array}{c}\text { RA offset } \\
(\mathrm{mas})\end{array}$ & $\begin{array}{c}\text { Dec offset } \\
(\mathrm{mas})\end{array}$ & $\begin{array}{c}S_{v} \\
(\mathrm{mJy})\end{array}$ & $\begin{array}{c}\text { Major axis } \\
(\mathrm{mas})\end{array}$ & Axis ratio & $\begin{array}{c}\text { PA } \\
\left({ }^{\circ}\right)\end{array}$ & $\begin{array}{c}T_{\mathrm{b}} \\
(\mathrm{K})\end{array}$ \\
\hline Uniform elliptical disk & 0 & 0 & $544.7 \pm 0.5$ & $57.8 \pm 0.1$ & $1.09 \pm 0.01$ & $53.5 \pm 0.4$ & $2760 \pm 140$ \\
Elliptical Gaussian (NE-peak) & $9.4 \pm 0.1$ & $12.9 \pm 0.1$ & $16.1 \pm 0.4$ & $19.8 \pm 0.5$ & $2.38 \pm 0.02$ & $159.3 \pm 1.1$ & $1055 \pm 90$ \\
Circular Gaussian (east-extension) & $27.0 \pm 0.1$ & $-3.6 \pm 0.1$ & $16.6 \pm 0.3$ & $8.9 \pm 0.2$ & 1 & $\ldots$ & $2260 \pm 135$ \\
Thin elliptical ring & 0 & 0 & $33.8 \pm 0.4$ & $70.0 \pm 0.2$ & $1.09 \pm 0.01$ & $53.5 \pm 0.4$ & $\ldots$ \\
\hline
\end{tabular}

Notes. Positive right ascension (RA) and declination (Dec) values represent east and north, respectively. $S_{v}$ is the flux density. Axis ratio $=$ major axis/minor axis. Position angle (PA) is measured east of north. $T_{\mathrm{b}}$ is the brightness temperature. Error values are the fitting errors except for the brightness temperature values which include the uncertainty in the absolute flux density.

of the east-extension in reality will be higher than this value because a portion of it lies on top of the uniform elliptical disk. We attribute the thin ring of emission beyond the uniform intensity disk as emission that is not accounted for in the idealized uniform disk model. The ring is not located close to the limb of the main disk which suggests that it is not a result of limb brightening. The lack of a detection of limb brightening means that the thermal gradients are small on the scale height being probed when the finite resolution is accounted for.

The continuum data have only $\sim 4 \%$ fractional bandwidth coverage but the high $\mathrm{S} / \mathrm{N}$ allows the spectral index, $\alpha$ (i.e., $S_{v} \propto v^{\alpha}$ ), of the emission to be constrained. We find the global spectral index to be $\alpha=1.67 \pm 0.09$ by separately imaging the lower (at $331.86 \mathrm{GHz}$ ) and upper (at $344.09 \mathrm{GHz}$ ) portions of the bandpass and calculating the integrated flux density in each image. This is slightly larger than the $\alpha=1.57 \pm 0.05$ that can be derived from previous unresolved millimeter observations (Altenhoff et al. 1994; Harper et al. 2009; O'Gorman et al. 2012) and is much larger than the $\alpha=1.33 \pm 0.01$ that can be derived from multi-epoch centimeter studies (O'Gorman et al. 2015a). We note that the NE-peak has a spectral index of $\lesssim 2$ in the spectral index image (from CASA's clean parameter $n$ terms $=2$ ) but the error values per pixel may be underestimated due to the small fractional bandwidth coverage. Both the global and pixel spectral indices are in agreement with the emission being thermal free-free in nature, while the increasing $\alpha$ towards higher frequencies is a manifestation of probing smaller density scale heights at higher frequencies.

\section{Discussion and conclusion}

\subsection{Verification of a temperature inversion between the photosphere and chromosphere}

Submm spatially resolved observations of thermal continuum emission from stellar atmospheres can act as an approximate linear thermometer. The continuum flux density, $S_{v}$, can be described as arising from an optically thick disk of angular size $\theta_{v}$ at some frequency $v$, and gas temperature $T_{\text {gas }}$ such that $S_{v} \propto T_{\text {gas }} \theta_{v}^{2}$. At these frequencies the free-free opacity varies as roughly $v^{2}$, so multi-frequency observations allow the temperature profile to be constructed. This method has been used by Lim et al. (1998) and O'Gorman et al. (2015a) to show that the mean gas temperature of Betelgeuse's extended atmosphere declines from approximately $3600 \mathrm{~K}$ at $2 R_{\star}$ to $1400 \mathrm{~K}$ at $6 R_{\star}$, and is not as hot $(\sim 8000 \mathrm{~K})$ as previously thought (e.g., Hartmann \& Avrett 1984). These values are plotted in Fig. 2 along with our ALMA measurement of $2760 \pm 140 \mathrm{~K}$ at $\sim 1.3 R_{\star}$. This value is below the photospheric effective temperature of $3690 \pm 54 \mathrm{~K}$ (Ohnaka et al. 2011) and most of the temperature

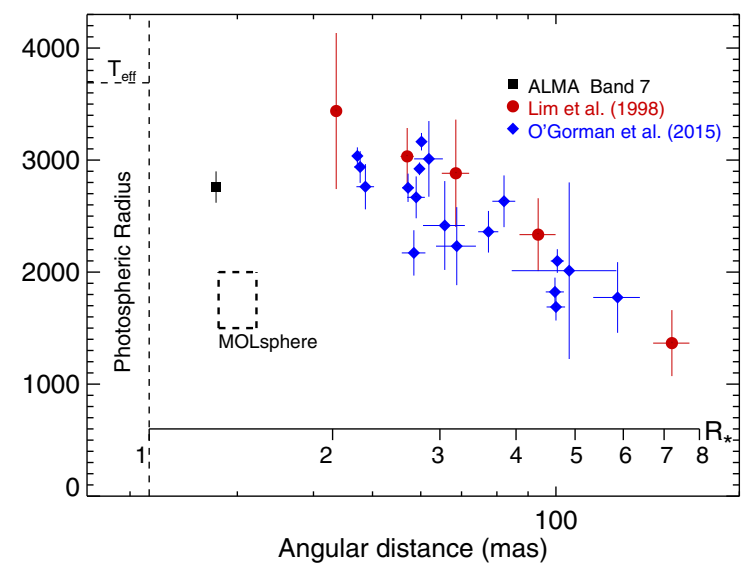

Fig. 2. Semi-log plot of the temperature profile of Betelgeuse's extended atmosphere. The red filled circles and blue filled diamonds represent the gas temperature derived from multi-epoch spatially resolved radio observations (Lim et al. 1998; O'Gorman et al. 2015a). The large black dashed rectangle is the approximate location and temperature range of the MOLsphere. The black filled square is our ALMA band 7 temperature which shows that the mean gas temperature has dropped well below the effective temperature at $\sim 1.3 R_{\star}$.

measurements between $\sim 2$ and $3 R_{\star}$. Obviously, there is not a monotonic decrease in the mean gas temperature of the extended atmosphere from the photosphere outwards. Indeed, modeling of infrared molecular lines from RSGs has already provided evidence for the presence of cool (1500-2000 K) gas between 1.3 and 1.5 $R_{\star}$ (Tsuji 2000; Perrin et al. 2004, 2007; Ohnaka 2004; Montargès et al. 2014). However, these models are not sensitive to hotter plasma that may be co-located with the cool gas. Our ALMA measurement provides the mean gas temperature and is the reason why our value at $\sim 1.3 R_{\star}$ is larger in comparison to MOLsphere values.

Lim et al. (1998) suggested that photospheric-like temperatures at small stellar radii (i.e., at $\sim 2 R_{\star}$ ), along with the monotonic decrease in temperature with increasing distance from the star, could be explained by the expansion and cooling of material elevated from the photosphere by large convection cells. In this scenario, the mean gas temperature should not drop below the effective temperature between 1 and $2 R_{\star}$, but we find that it does. Our finding is in agreement with chromospheric modeling of all cool stars, which suggests that there is a trend of decreasing gas temperature above the photosphere which then rises again into the chromosphere (e.g., Basri et al. 1981).

\subsection{The source of the submm continuum asymmetries}

At the depths probed by the $338 \mathrm{GHz}$ emission the gas temperature will vary much more slowly with height above the surface, $z$, 
than the hydrogen $\left(n_{\mathrm{H}}\right)$ and electron $\left(n_{\mathrm{e}}\right)$ densities, respectively (Basri et al. 1981). Eddington-Barbier relations show that for temperatures varying slowly with optical depth $T_{\mathrm{b}} \simeq T_{\text {gas }}(\tau=1)$, where

$\tau(z) \propto \int_{z}^{\infty} n_{\mathrm{e}} n_{\mathrm{H}} \mathrm{d} z^{\prime} \simeq n_{\mathrm{e}}^{2}(z) H_{\rho} / 2$

where $H_{\rho}$ is the density scale height. The latter equality follows if both $n_{\mathrm{e}} / n_{\mathrm{H}}$ and $H_{\rho}$ are approximately constant. This demonstrates that the submm opacity is dominated by the local densities and the local scale-height. Localized (i.e., non-uniform) heating will not only increase the gas temperature but it will also increase $n_{\mathrm{e}}$ (from photoionized metals), the density scale-height, and thus the total optical depth. This could explain why the NEpeak is the brightest feature in our image of Betelgeuse. On the stellar disk we are sampling $T_{\text {gas }}=2760 \mathrm{~K}$, but if the temperature increases outwards as a result of local heating, then the $\tau=1$ surface moves to a greater height where $T_{\text {gas }}=3815 \mathrm{~K}$. The eastextension can then be explained by the same phenomenon but only observed on the limb.

Uitenbroek et al. (1998) tentatively associated a strong UV hot spot with the location of the stellar rotation pole. Uitenbroek et al. (1998) and Dupree (2011) suggested that global shock waves generated by pulsations would be more intense at the pole because of the steeper density gradients caused by stellar rotation. They suggested this scenario as the heating mechanism behind their detected UV hot spots. However, our detected regions of localized heating do not coincide with the UV hot spot, making the physical association with the pole less likely.

Solar submm observations probe the lower chromosphere immediately above the temperature minimum. Applying the opacity scaling laws from Ayres (1979) to Betelgeuse shows that its chromosphere has a lower opacity than the Sun and so our ALMA observations are most likely probing very close to the temperature minimum. Solar submm studies show brightness temperature fluctuations across the solar disk with values $\sim 1000 \mathrm{~K}$ in excess of the quiet Sun brightness in regions of strong magnetic field strength (Lindsey et al. 1995; Lindsey \& Kopp 1995). Given that Betelgeuse has a longitudinal magnetic field strength of $\sim 1 \mathrm{G}$ (Aurière et al. 2010), which is similar to the solar value, and it is predicted to harbor local regions of strong magnetic fields (Dorch 2004), it is possible that the submm asymmetries are related to magnetic activity. Near-infrared interferometric observations (e.g., Montargès et al. 2016; Haubois et al. 2009) suggest there are large convection cells on the surface of Betelgeuse. Dorch (2004) carried out 3D convection simulations for Betelgeuse and showed that these cells could sustain a local magnetic field. Indeed, a bright spot linked to a large convection cell has been reported in the north-east quadrant of Betelgeuse's photosphere less than one month prior to our ALMA observations (Tessore et al. 2017), which is tantalizing evidence for a link between photospheric convection and heating in the atmosphere of a RSG.

Our suggestion that the detected localized heating is due to magnetic activity would be in agreement with the conclusion in Harper \& Brown (2006) that very thin "filamentary" structures are required so that the chromospheric component is not completely opaque and detectable atcm wavelengths. These filaments would have a very small filling factor but could have gas temperatures similar to those implied by UV chromospheric continuum and lines studies (i.e., $T_{\text {gas }} \sim 8000 \mathrm{~K}$ ). However, our limited spatial resolution would smear out these fine structures and would reduce the contrast in the brightness temperatures. Indeed, Loukitcheva et al. (2015) have shown that this will happen when active regions on the Sun are observed at low spatial resolution with ALMA. Future multi-frequency ALMA observations with similar spatial resolution to that presented here could confirm our hypothesis if the brightness temperatures of the asymmetries increase with decreasing frequency as a result of the shifting of contributing heights to higher and hotter layers in the atmosphere.

Acknowledgements. This paper makes use of the following ALMA data: ADS/JAO.ALMA\#2015.1.00206.S. ALMA is a partnership of ESO (representing its member states), NSF (USA) and NINS (Japan), together with NRC (Canada), NSC and ASIAA (Taiwan), and KASI (Republic of Korea), in cooperation with the Republic of Chile. The Joint ALMA Observatory is operated by ESO, AUI/NRAO and NAOJ. We thank the Nordic ALMA Node for providing computational resources for this project. E.O.G. acknowledges support from the Irish Research Council. We acknowledge financial support from the "Programme National de Physique Stellaire" (PNPS) of CNRS/INSU, France. G.M.H. received support from HST grant HST -AR-14566.001-A which was provided by NASA through a grant from the Space Telescope Science Institute, which is operated by the Association of Universities for Research Astronomy, Incorporated, under NASA contract NAS5-26555.

\section{References}

Altenhoff, W. J., Thum, C., \& Wendker, H. J. 1994, A\&A, 281, 161 Aurière, M., Donati, J.-F., Konstantinova-Antova, R., et al. 2010, A\&A, 516, L2

Ayres, T. R. 1979, ApJ, 228, 509

Basri, G. S., Eriksson, K., \& Linsky, J. L. 1981, ApJ, 251, 162

Dorch, S. B. F. 2004, A\&A, 423, 1101

Dupree, A. K. 2011, in Physics of Sun and Star Spots, eds. D. Prasad Choudhary, \& K. G. Strassmeier, IAU Symp., 273, 188

Gilliland, R. L., \& Dupree, A. K. 1996, ApJ, 463, L29

Harper, G. M., \& Brown, A. 2006, ApJ, 646, 1179

Harper, G. M., Brown, A., \& Lim, J. 2001, ApJ, 551, 1073

Harper, G. M., Richter, M. J., Ryde, N., et al. 2009, ApJ, 701, 1464

Harper, G. M., Brown, A., Guinan, E. F., et al. 2017, AJ, in press

Hartmann, L., \& Avrett, E. H. 1984, ApJ, 284, 238

Hartmann, L., \& MacGregor, K. B. 1980, ApJ, 242, 260

Haubois, X., Perrin, G., Lacour, S., et al. 2009, A\&A, 508, 923

Holzer, T. E., \& MacGregor, K. B. 1985, in Mass Loss from Red Giants, eds.

M. Morris \& B. Zuckerman, Astrophys. Space Sci. Lib., 117, 229

Holzer, T. E., Fla, T., \& Leer, E. 1983, ApJ, 275, 808

Kervella, P., Lagadec, E., Montargès, M., et al. 2016, A\&A, 585, A28

Lim, J., Carilli, C. L., White, S. M., Beasley, A. J., \& Marson, R. G. 1998, Nature, 392, 575

Lindsey, C., \& Kopp, G. 1995, ApJ, 453, 517

Lindsey, C., Kopp, G., Clark, T. A., \& Watt, G. 1995, ApJ, 453, 511

Loukitcheva, M., Solanki, S. K., Carlsson, M., \& White, S. M. 2015, A\&A, 575, A15

Martí-Vidal, I., Vlemmings, W. H. T., Muller, S., \& Casey, S. 2014, A\&A, 563, A136

Montargès, M., Kervella, P., Perrin, G., et al. 2014, A\&A, 572, A17

Montargès, M., Kervella, P., Perrin, G., et al. 2016, A\&A, 588, A130

O'Gorman, E., Harper, G. M., Brown, J. M., et al. 2012, AJ, 144, 36

O'Gorman, E., Harper, G. M., Brown, A., et al. 2015a, A\&A, 580, A101

O'Gorman, E., Vlemmings, W., Richards, A. M. S., et al. 2015b, A\&A, 573, L1

Ohnaka, K. 2004, A\&A, 421, 1149

Ohnaka, K., Weigelt, G., Millour, F., et al. 2011, A\&A, 529, A163

Perrin, G., Ridgway, S. T., Coudé du Foresto, V., et al. 2004, A\&A, 418, 675

Perrin, G., Verhoelst, T., Ridgway, S. T., et al. 2007, A\&A, 474, 599

Tessore, B., Lòpez-Ariste, A., Mathias, P., et al. 2017, ArXiv e-prints

[arXiv: 1702.02002]

Tsuji, T. 2000, ApJ, 538, 801

Uitenbroek, H., Dupree, A. K., \& Gilliland, R. L. 1998, AJ, 116, 2501 


\section{Appendix A: Continuum imaging}

Continuum imaging was performed using the multi-frequency synthesis CLEAN algorithm with Briggs weighting and a robust parameter of 0 , which resulted in a synthetic beam size of $15 \times 13$ mas at $338 \mathrm{GHz}$. Two Taylor coefficients were used to model the linear frequency dependence of the continuum emission. Additionally, we used the multiscale imaging option with scales approximately corresponding to 0,1 , and $3 \times$ the synthesized beam. Using these imaging parameters, three iterations of phase-only self-calibration were performed on the continuum emission until a $\mathrm{S} / \mathrm{N}$ convergence was reached with a solution interval of $15 \mathrm{~s}$. One final iteration was then performed, solving for both amplitude and phase. We note that self-calibrating the continuum data reduced the rms noise by approximately an order of magnitude. To investigate small-scale structure in the continuum emission we also created images using Briggs weighting with a robust parameter of -1 and uniform weighting. The achieved rms noise for the continuum images was $85 \mu \mathrm{Jy}_{\text {beam }^{-1}}$ (robust 0 images), $138 \mu \mathrm{Jy}_{\text {beam }}{ }^{-1}$ (robust $-1 \mathrm{im}$ ages), and $255 \mu \mathrm{Jy} \mathrm{beam}^{-1}$ (uniform images).

\section{Appendix B: Residual images from uv-fitting}

In Fig. B.1 we show three example residual images from our $u v$-fitting analysis. Residual images containing a lot of emission meant that the subtracted model did not describe the data well. In the left panel of Fig. B.1 a uniform intensity elliptical disk was fitted to the visibilities. The best fit model was then subtracted from them using CASA's $u v s u b$ task, and the residual visibilities were then imaged. The rms of the residual image within a circle of radius $\sim 3 R_{\star}$ centered on the disk center was
$1.345 \mathrm{mJy}$ beam $^{-1}$. Clearly, excess emission remains in the residual image: a strong $\left(\sim \pm 60 \sigma_{\text {rms }}\right)$ elongated feature in the northeast quadrant, another strong $\left(\sim \pm 40 \sigma_{\text {rms }}\right)$ feature east of the disk center, and a weaker $(\sim \pm 10 \sigma)$ ring-like feature beyond these. In the middle panel of Fig. B.1 we show the residual image after the best fit model of a uniform intensity elliptical disk plus a Gaussian profile have been fitted and subtracted from the visibilities. The emission feature in the north-east quadrant is now gone and the rms of the residual image within a circle of radius $\sim 3 R_{\star}$ centered on the disk center was $0.817 \mathrm{mJy} \mathrm{beam}^{-1}$. Finally, in the right panel of Fig. B.1 we show the residual image after the best fit model of a uniform intensity elliptical disk plus two Gaussian profiles plus a thin ring have been fitted and subtracted from the visibilities. All the main emission features present in the left panel of Fig. B.1 are now gone and the rms of the residual image within a circle of radius $\sim 3 R_{\star}$ centered on the disk center was $0.367 \mathrm{mJy}^{\text {beam }}{ }^{-1}$. We note that a number of small-scale features with $\left(\sim \pm 5-10 \sigma_{\text {rms }}\right)$ significance remain, which are probably the result of the thin ring not being a perfect match for the low brightness emission beyond the main elliptical disk.

We found that replacing the elliptical uniform intensity disk with a circular uniform intensity disk in the multi-component fits described previously produced larger chi-squared statistic values and increased the residual image rms. We also attempted fits by forcing the Gaussian components to have negative flux density values, but the fitted values were smaller than the fitting errors. Moreover, the large negative features in the left and middle panel are not present in our best fit model shown in the right panel and we conclude that they are simply artefacts.
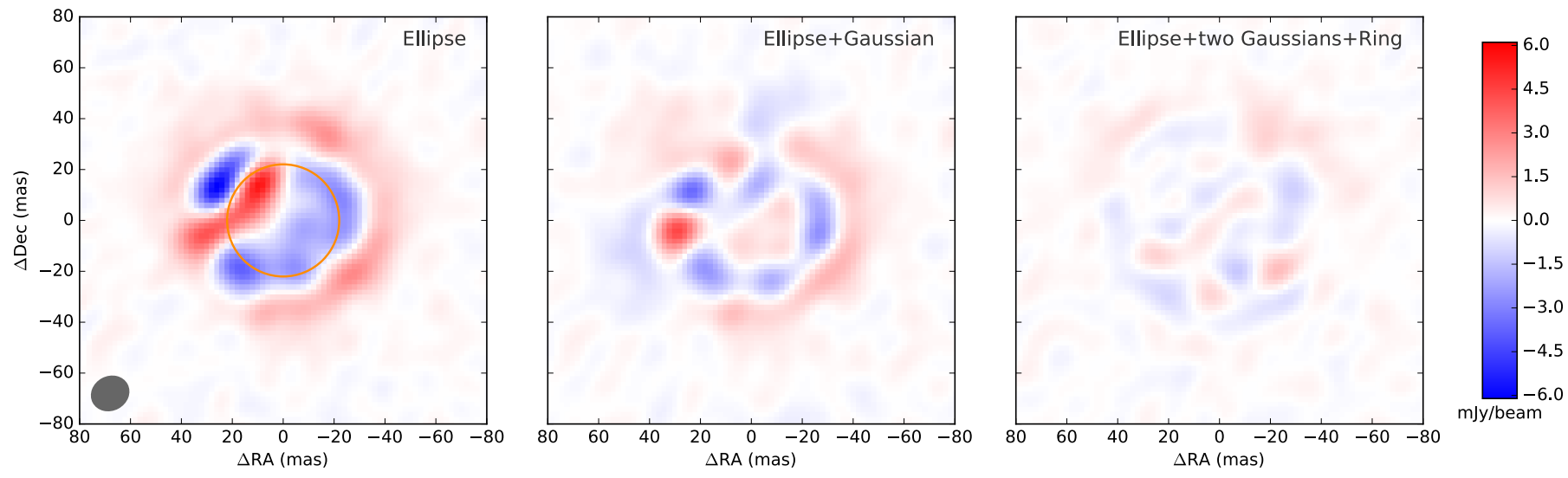

Fig. B.1. ALMA $338 \mathrm{GHz}$ continuum residual (i.e., data - model) images of Betelgeuse. The filled ellipse in the lower left corner of the left panel is the FWHM of the restoring beam and has dimensions $15 \times 13$ mas while the orange circle is the size of the infrared $H$-band photosphere from Montargès et al. (2016). Left panel: the residual image after a uniform elliptical disk has been subtracted from the visibilities. Middle panel: the residual image after a uniform elliptical disk plus an elliptical Gaussian have been subtracted from the visibilities. Right panel: the residual image after a uniform elliptical disk, an elliptical Gaussian, a circular Gaussian, and a thin ring have been subtracted from the visibilities. 